\title{
The Everyday lives and coping Strategies of women in Delhi; Discerning roots of Psychological Empowerment: An Ethnographic Study
}

\author{
Swati Sharma $^{1}$, Dr. Anita Gupta ${ }^{2}$
}

\section{ABSTRACT}

This is an ethnographic study based on inductive reasoning inspired by everyday life coping of slum women in Delhi. Evidences from field work were found to be congruent with the underlying assumptions of Humanistic and analytical psychology, perspectives which ascertain positive human values and life orientation such as growth, and fulfillment, making it difficult to completely neglect strategies for adaptability, positive coping, and adjustment, which are having a positive influence in everyday lives of slum women in their day-day life. The focus of this study is not to explore the daily life concerns, but to highlight how these concerns are addressed by slum women, with regard to their coping strategies. The objective of this study was to bring into light the phenomenon of positive adaptability towards daily life concerns, in context with slum women by exploring three coping strategies given by Endler and Parker. Task-oriented strategy, Emotion-oriented strategy, and, Avoidance-oriented strategy three categories which were used to categorize the responses towards daily life stressors. Finally this study attempts to fill in the prevailing literature gap in the context of the conceptualization of psychological empowerment for slum women based on the findings of this study and trace the roots of psychological empowerment using the perspectives of psychology. This study illustrates analysis of ethnographic records of 50 informants from various slums in Delhi.

Keywords: Psychological, Empowerment, Slum, Women, Coping Strategy

Sociologists and psychologists in the past have worked extensively in the area of women empowerment. Literature in terms of strategies used for empowerment of women is enormous too. Also, mental health care services, their unavailability, and the prevailing psychopathology in slum, poor living conditions and their daily life concerns has also been examined by literature, to

\footnotetext{
${ }^{1}$ Research scholar, Faculty of Management studies, Manav Rachna International University, Faridabad, India

${ }^{2}$ Professor, Faculty of Management studies, Manav Rachna International University, Faridabad, India

*Responding Author

Received: March 15, 2017; Revision Received: March 29, 2017; Accepted: March 30, 2017

(C) 2017 Sharma S, Gupta A; licensee IJIP. This is an Open Access Research distributed under the terms of the Creative Commons Attribution License (www.creativecommons.org/licenses/by/2.0), which permits unrestricted use, distribution, and reproduction in any Medium, provided the original work is properly cited.
} 


\section{The Everyday lives and coping Strategies of women in Delhi; Discerning roots of Psychological Empowerment- An Ethnographic Study}

a great extent. But unfortunately, not enough literature is available in the context of coping mechanisms of slum women in their everyday lives. It is vital to accept that the phenomenon of abnormality runs analogous with 'normality'. The available literature in this context is only suggestive of 'what the problem' is and not on 'how is the problem solved'. Keeping this into consideration, this study focuses not on the daily life concerns and psychopathology, but aims to have an in-depth understanding on the positive aspects of coping strategies, which are contributing in positive adjustment and coping in everyday lives of the slum women in Delhi.

This study is based on the process of inductive reasoning, wherein field observations of everyday lives Slum and, an understanding in context with coping strategies were observed to be symbolic of strategies which enable them for better adaptability and positive coping based on the earlier perspectives of psychology. The themes were observed to be congruent with perspectives of psychology, which were suggestive of positive growth, self actualization, manifestation of characteristics of both masculine, and feminine gender roles by slum women (regardless of natural biological sex). In the light of the assumptions of humanistic perspective, and analytical psychology, and evidences from everyday life coping, it becomes difficult to simply focus on literature that is suggestive of psychopathology, and lack of resources for a livelihood. It is also essential to note that, the unavailability of resources is an external factor for the informant, whereas coping abilities are dependent on internal factors, which are essential parameters, when one is trying to understand the extent of adaptability and positive coping in everyday life.

Coping is a dynamic situation specific reaction to stress. It is a set of concrete responses to stressful situations or events that are indented to resolve the problem and reduce stress which indicate towards the psychological effectiveness and efficiency of an organism to deal with daily life concerns and maintain equilibrium after overcoming the prevailing stressor. Such a state can also be viewed as psychologically empowering state, which facilitates better adaptability and adjustment to daily life stressors.

In order to explore the answer to the research question, an ethnographic study was carried out in Delhi slums. The objective of this study was to bring into light the phenomenon of positive adaptability towards daily life concerns, in context with slum women by exploring three coping strategies given by Endler and Parker. Task-oriented strategy, Emotion-oriented strategy, and, Avoidance-oriented strategy, are the three categories, which were used to categorize the responses of the participants towards daily life stressors. Finally this study attempts to fill in the prevailing literature gap in the context of the conceptualization of psychological empowerment for slum women based on the findings of this study and trace the roots of psychological empowerment using the perspectives of psychology. This study maintains its ecological validity by conducting it in a naturalistic setting to achieve the following objectives- 
The Everyday lives and coping Strategies of women in Delhi; Discerning roots of Psychological Empowerment- An Ethnographic Study

\section{Objectives Of The Study}

- To critically examine psychological perspectives and explore how they relate with psychological empowerment for slum women in their everyday lives.

- To explore the extent to which the themes and findings of the study relate to the psychological perspectives and psychological empowerment.

- To fill in literature gap in terms of understanding how psychological empowerment can be viewed in the everyday lives of slum women residing in Delhi.

\section{METHODOLOGY AND DATA COLLECTION}

The design of the study is Ethnographic. Intensive field observations, informal conversations with the informants, open ended interviews and ethnographic daily field records are significant in concluding the findings of this study. Its qualitative design and a nature to maintain its ecological validity, and an attempt to have an understanding of everyday life interactions of slum women residing in Delhi have facilitated the accomplishment of its objectives. This study attempts to suggest its findings based on ethnographic records of 50 informants, who are residents of slum in Delhi. Data has been collected from various locations of Delhi, to have an exhaustive account of the everyday lives of these women living in different slums. Finally Narrative analysis was adopted in order to suggest the significant findings of the study.

The ethnographic field work was conducted in various phases with each informant. Following are the phases of data collection which aimed at collecting a comprehensive overview in context with, each informant's values, attitudes, and assumptions, and their coping in everyday lives-

- Naturalistic observation and rapport formation with informants

- Slum tour

- Conduction of interview questionnaire- genological chart, family tree, reflection of kinship system, grand tour questions, mini tour questions, experience questions, hypothetical interaction questions.

- Categorization of coping strategies as- avoidance oriented, emotion oriented strategy, taskoriented strategy based on analysis. 
The Everyday lives and coping Strategies of women in Delhi; Discerning roots of Psychological Empowerment- An Ethnographic Study

1. Date:

2. Time:

3. Location:

4. Name:

5. Age:

6. Cast:

7. Family type:

8. Position in family:

9. FAMILY TREE
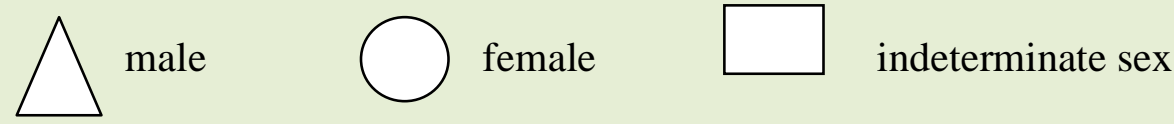

$=$ Divorce $/$ marriage $/$ Deceased individual

10.

e- ego

individual in concern is referred to as "ego" and an ' $e$ ' (for ego) indicates the position of that individual at the centre of the kinship chart

11. Refection of Kinship system- bought up of offspring and childhood

12. Interview responses

13. Observation and analysis of coping strategy- based on interview responses

14. Aspects of non verbal communication

Figure 1: Sample response card used for data collection

\section{Sampling}

Purposive sampling

The criteria for choosing participants will be on the basis of the following considerations

- $\quad$ She is the primary caregiver for her family

- $\quad$ She is a resident of Delhi or Delhi NCR slum area

- $\quad$ She is not acquainted with formal or informal education

- $\quad$ She is of age group 20-30 years

\section{LITERATURE REVIEW AND LITERATURE GAP}

Psychological empowerment defined from the perspective of the individual employee by literature

Psychological empowerment according to Spretizer (1995 a) has been defined as a motivational construct manifested in four cognitions: meaning, competence, self determination, and impact which reflect an active orientation to a work role where the individual wishes and feels that he or she is able to shape his/her work role or context. Another study by Menon and Sanjay (1999)

(C) The International Journal of Indian Psychology, ISSN 2348-5396 (e)| ISSN: 2349-3429 (p) | 161 
The Everyday lives and coping Strategies of women in Delhi; Discerning roots of Psychological Empowerment- An Ethnographic Study

defined psychological empowerment from the perspective of the individual employee, wherein a psychologically empowered state was considered to be a cognitive state characterized by a sense of perceived control, perceptions of competence, and internalization of goals and objectives of the organization. These studies however, gained an in-sight to the understanding of the concept of psychological empowerment and its conceptualization for employees and the working population, but literature is limited in terms of understanding and conceptualization of this concept for the slum population in our country.

Focus of literature on challenges in mental health care and the prevalence of psychological and biological concerns in the past for slum women, and not on effective coping mechanisms

Anant kumar 2011, reflected upon the challenges and prospects of mental health services in India, wherein he has emphasized on the lack of trained human resource for mental health care, and the lack of political commitment, and realization that mental health is an important aspect of our health system.

Study by Srinivas Murthy, 2010, highlighted the distribution of mental health services in India, at, Primary, secondary, and Tertiary level including rehabilitation.

Other research work done in the area suggests the prevailing abnormality statistics; some works illustrate biological health of women in slum areas, and others focused on the inaccessibility of mental health care and its concerned professionals for slum areas but, unfortunately, there is not much research on psychological empowerment of slum women in this area.

Literature gap prevails in context of conceptualizing cognitions which are facilitating adaptive functioning for slum women in their everyday lives

Psycho-social research has investigated psychopathology for years and has focused on the prevailing abnormality statistics for slum women. But, the recognition and receptivity towards phenomenon which are symbolic of effective and positive cognition, which enable them to adapt and efficiently function in environment, and make appropriate decisions and choices to deal with everyday life stressors, has not been given much attention. This is resulting in literature gap, in terms of conceptualizing cognitions which are aiding adaptive functioning for slum women, despite the absence, of sufficient resources to facilitate survival and effective coping in their everyday lives.

Psychopathology and psychological empowerment run analogous with each other in context with everyday lives of slum women

One must make a note that, the concept of psychopathology runs parallel with overt and covert responses which are symbolic of effective coping in everyday lives of slum women. This view

(c) The International Journal of Indian Psychology, ISSN 2348-5396 (e) | ISSN: 2349-3429 (p) | 162 


\section{The Everyday lives and coping Strategies of women in Delhi; Discerning roots of Psychological Empowerment- An Ethnographic Study}

calls for thought that if there is psychopathology shaping in slums due to lack in availability of resources and appropriate living conditions, resulting in shortage of proper facilities for health, and sanitation, lack of drainage, lack of medical facilities, prevalence of psychological disorders, and other medical concerns due to shortage of mental and physical health care, there is also, effective coping, which can be observed in everyday lives of slum women. Taking into consideration the drive for everyday survival, need to make appropriate decisions, high motivation, and experience of positive emotion, perception of a positive self and social identity , identity of a primary care giver, and one's ability to carry out these roles, development of interpersonal relationships for a good social life, the ability to choose the best alternative considering the long term impact on their lives, and many other factors enabling positive coping and daily survival, one may view that, despite unavailability and lack of appropriate knowledge and resources, these women residing in slums located in Delhi , to a great extent are manifesting responses, which may be viewed as a psychologically empowered response, as , "Psychological empowerment can be defined as any phenomenon over a lifespan which enables an individual to adapt to any existing environmental stressor, and respond to it, considering the overall long term reimbursement of his/her current decision making.

According to this, research in its futuristic vision must also focus on cognitions and behaviors which make an individual adaptable, well-adjusted in cultures, such as slums wherein , the conditions for survival and psychological empowerment are even more challenging, considering the unavailability of resources. , but unfortunately literature is very limited in the context of studies which focus on behaviors which focus on effective coping, positive adjustment, towards everyday life stressors and challenges. Moreover, psychological empowerment has still been defined in the organization sector, and from the point of view of an employee in a particular organization in literature, but such a concept is yet to be investigated, recognized, and furthermore be conceptualized particularly in the context of slum culture, in order to have an indepth understanding of values, attitudes, and assumptions, which are facilitating positive coping among individuals.

\section{CONCLUDING REFLECTIONS}

1. Discerning psychological empowerment within the roots of psychological perspectives

\subsection{Tracing Psychological empowerment in the eyes of Analytical psychology}

According to Jung, for achieving unity and wholeness, a person must become increasing aware of the wisdom available in one's personal and collective unconscious, and must learn to live in harmony with it. He affirms that many of modern life problems in everyday lives are caused by "mans progressive alienation from his instinctual foundation. Jung argues that, universal archetypes are products of collective experience of men and women living together. In modern western civilization men are discouraged from living their feminine side, and women from experiencing their masculine tendencies. Psychological development according to him was 


\section{The Everyday lives and coping Strategies of women in Delhi; Discerning roots of Psychological Empowerment- An Ethnographic Study}

undermined due to lack of expression in overt and covert responses, exhibiting masculine tendencies for females, and famine tendencies for males. This may suggest that more an individual is aware of his instinctual foundation, and engages in behaviors exhibiting his/her conscious mind, irrespective of their specific gender roles in their everyday lives, which comprises of the thought, emotions, and, memories a person is aware of, the more the possibility for positive psychological development, wholeness, positive adaptation, and effective coping in everyday life can be anticipated.

Also, Jung's emphasis on the competing forces and structures within the individual that must be balanced rather than between the individual, , and demands of the society, or between the individual and reality, point towards another significant explanation that inspires the finding of this study, which explains that external stressors will have little or least control over effective coping in everyday life, if an individual is balanced in context with the manifestation of his/ego , and conscious mind, regardless of his/her gender roles and expression of masculine and famine tendencies.

Furthermore, many informants in the course of this study have been observed to be taking on the roles of the opposite gender, such as role of a financial caregiver in case of demise of their spouse, independent living, enjoying single parenthood, and assuming the role of a father figure, working as labors in case their husband lost their jobs, in other cases unemployed, as well as assuming their natural role of being a primary care givers to the family, maintaining their individual state of selfhood.

Finally, one can suggest that cognitive and behavioral responses, manifested in terms of coping strategies, which are facilitated by a conscious understanding of the long term impact of one's current decision making in everyday lives, maintaining a state of selfhood and balance, irrespective of the expression of a natural gender role, may be anticipated to be a psychologically empowered response, ultimately facilitating better adaptation and survival in everyday life.

\subsection{Behaviorism and the everyday lives of slum women}

For most behaviorists, the structural unit of Personality is the response. Each response is behavior, which is emitted to satisfy a specific need .This implies that, one can study an individual's attitudes, beliefs, values, and assumptions by studying the type of responses an individual gives, to deal with daily life stressors. Moreover, Psychology as a study of behavioral science examines the study of behavioral responses, both overt and covert. As John Watson explains, psychology is the study of behavior or responses (to stimuli) which can be measured and studied objectively. This implies that, not only, one can examine overt and covert responses towards daily life problems to examine the nature of coping and adjustment but also, study 


\section{The Everyday lives and coping Strategies of women in Delhi; Discerning roots of Psychological Empowerment- An Ethnographic Study}

objectively the manifestation of the thinking patterns in coping strategies of slum women in their everyday life.

According to the operational definition, Psychological empowerment can be defined as any phenomenon over a lifespan which enables an individual to adapt to any existing environmental stressor, and respond to it, considering the overall long term reimbursement of his/her current decision making." Henceforth, responses to environmental stimulation, which are in procession with adaptability, and based on a vision for future positive reimbursement of current decision making, may be symbolic of psychological empowerment.

Based upon the perspective, that psychology is a scientific study of human behavior, this study was designed to examine the nature of coping strategies, considering that, responses of the informants in their everyday life to stressors will represent their respective thinking patterns and schemas. Influence of behaviorism and objective study of responses, facilitated the natural orientation of this study, which enabled clarity to view attitudes, assumptions and behaviors of the informants, in an entirely different way. Hence it became easier to establish that, the everyday lives of the slum women residing in Delhi, are much beyond the statistics of the prevailing abnormalities, and, concerns of basic necessities.

It is also crucial to state here that, psychopathology in the slums does prevail, but with that, effective coping and positive adjustment also prevails in the everyday lives of slum women, and with these effective coping mechanisms, and well thought over responses, one may be able to trace a psychologically empowered response.

\subsection{Reflecting psychological empowerment in the eyes of Humanistic Approach}

Rogers and Maslow suggested the idea of a fully functioning person. Rogers believed that the fulfillment is the motivating force for personality development. People try to express their capabilities, potentials, and talents to fullest extent possible. There is an inborn tendency to maximize self concept through self actualization. In this process the self grows, and expands, and becomes more social. In this view, human beings are free to shape their lives, and to self actualize.

It is possible to view self actualization by analyzing the motivations that govern our life. In the context of this study, it is possible to reflect this state by studying responses which highlighted the characteristics of a person, who is self aware, socially responsive, creative in problem

solving, spontaneous in facing everyday life stressors, open to novelty, and challenge, depicts a sense of humor, and capacity for deep interpersonal relationships. 


\section{The Everyday lives and coping Strategies of women in Delhi; Discerning roots of Psychological Empowerment- An Ethnographic Study}

Friendships, self confidence, participation in community functions and celebrations, sense of excitement for the same, and withstanding daily life concerns, solving problems by coping strategies, for fulfillment, positive adaptation, and a focus on the existing environmental stressor for effective coping, has placed the informants on the path for self growth and development. This indicates that, the everyday life of women in the context of this study is so much more just than, biological needs.

It can be established that positive personality development, and the journey to optimally use one's psychological resources for coping in everyday life, will have a significant impact on the ability of an individual to make, psychologically empowered decisions. Moreover, observations from this study clearly indicate that, the more an informant was aligned with her self-concept, and optimal fulfillment of her potential in day to day life, the more she was content with her life. Such informants, during the process of the study were seen to apply more action-oriented strategies to solve their daily problems. Informants from okhla modh have reported to be going to schools and figure out a way wherein, their children could get better education. Other set of results from kusum pur pahadi highlighted the significance of going out with their friends for self time and good interpersonal relationships.

Finally one can establish that, the informants in the course of this study occupy higher needs, than simply physiological needs. Therefore, the path of self growth, development, and the free will to make choices, considering their long term impacts will facilitate their ability to make psychologically empowered decision sand responses in the future.

\section{Psychological empowerment, and its relationship with humanistic approach, behaviorism, and analytical psychology \\ 2.1 Psychological empowerment in the vision of this study}

"Psychological empowerment can be defined as any phenomenon over a lifespan which enables an individual to adapt to any existing environmental stressor, and respond to it, considering the overall long term reimbursement of his/her current decision making. Psychological empowerment in its vast context can be understood under the following nine parameters. i)a process of psychological advancement-, ii) a transformation within an individual for betterment, iii)a psychological state enabling change and encouraging mental adaptation, iii)a psychological enlargement of the possible opportunities in order to excel , in context to one's own interest, iv)an evolution of an individual's personality to withstand life's situations independently, positively, and firmly, v) expansion of own potentialities, extension and development of own psychological resources, vi) psychological furtherance to have a positive vision irrespective of the situations and life circumstances, gain of commitment confidence, and discipline towards one's goals, vii) the ongoing growth towards self development, improvement, progress in context with psychological maturity, viii) promotion of and assistance to the needy, ix) and finally the

(c) The International Journal of Indian Psychology, ISSN 2348-5396 (e)| ISSN: 2349-3429 (p) | 166 
The Everyday lives and coping Strategies of women in Delhi; Discerning roots of Psychological Empowerment- An Ethnographic Study

display of regeneration of all these factors and constant reinforcement of the interplay of all these factors in the members of the society."

2.2 Psychological empowerment and its relationship with behaviorism, analytical psychology and humanistic psychology

According to the data analysis 6 out of 10 elements are congruent to perspectives in psychology which affirm an individual's position on the path of self growth, effective coping in everyday life and adaptability to everyday life stressors. Hence the manifestation of these elements and their consistent interaction with each other will facilitate the process of psychological empowerment in everyday life.

The table below indicates the elements of psychological development and the psychological perspective it is based upon-

\section{Elements of psychological empowerment Psychological perspective basis}

1. Psychological furtherance

Humanistic

2. Self growth and development Humanistic

3. Awareness of opportunities for psychological

Humanistic

4. enlargement

5. Mental adaptation

behaviorism

Transformation, modification, and learning of

6. responses for effective coping.

7. Positive self monitoring and regulation Humanistic

8. Compassion towards other community members

Behavioral responses demonstrating Pro-social

9. behavior, promotion and assistance of fellow -

10. community members regardless of gender roles Regeneration of all elements of psychological development.

Contextual and appropriate application of cognitive resources for efficient coping in everyday lives.

\section{Figure 2- Relationship of psychological empowerment and psychological perspectives}

\subsection{The manifestation of psychological empowerment of slum women at a Psycho-social level} Menon and Sanjay T. (1999) defined psychological empowerment from the perspective of the individual employee. The psychologically empowered state was considered to be a cognitive state characterized by a sense of perceived control, perceptions of competence, and the 
The Everyday lives and coping Strategies of women in Delhi; Discerning roots of Psychological Empowerment- An Ethnographic Study

internalization of goals and objectives of the organization. i) At a psycho-social level this can be understood as a cognitive state characterized by a perceived control with slum women in the context of her social, ideal and relational self, which is reflected at a cognitive and behavioral context, when she takes on her roles of differentiated selves at a domestic and occupational level. ii) Perceptions of competence which is reflected in initiative taking in the context of full optimization of her skills and potentials, and even in terms of marketing her skills to earn a livelihood. iii) Finally, internalization of goals and objectives of the organization, which at a psycho-social level manifest in the form of internalization of goals and objectives which are crucial for day-day adaptive functioning and fulfillment of needs that are both individual communities, and culture specific.

The question which arises now is that, to what extent it is appropriate to claim that slum women are in the requirement for psychological empowerment, or is still in a process where they have to be equipped with skills which enable psychological empowerment, especially in the presence of evidence, that slum women are already psychologically empowered, which is apparent when manifestations of psychological empowerment can be observed, both, at a cognitive as well as behavioral level?

\section{Acknowledgments}

The author appreciates all those who participated in the study and helped to facilitate the research process.

\section{Conflict of Interests}

The author declared no conflict of interests.

\section{REFERENCES}

Endler, N. S., \& Parker, J. D. A. (1990). "Multidimensional Assessment of Coping: A Critical Evaluation.” Journal of Personality and Social Psychology, 58(5), 844-854.

Sharma, S.W and Gupta, A. N. Faculty of management studies. Manav Rachna International University. Faridabad. (2016). Reading between the lives of slum women in Delhi. Unpublished manuscript.

Sharma,S.W and Gupta , A.N . Faculty of Management studies. Manav Rachna International University. Faridabad. (2016). The fundamentals of psychological empowerment and its elements; a perspective from everyday lives of slum women. Unpublished manuscript.

Sanjay, M.T. (1999) Psychological Empowerment: Definition, Measurement, and Validation. Canadian Journal of Behavioural Science

Menon, Sanjay T (1999). Psychological Empowerment: definition, measurement, and validation. Canadian Journal of Behavioural Science. 
The Everyday lives and coping Strategies of women in Delhi; Discerning roots of Psychological Empowerment- An Ethnographic Study

Oladipo, SE. Psychological empowerment and development. Edo journal of counseling. Vol(2). 2009

Patel V, et al. Women, poverty and common mental disorders in four restructuring societies. Soc Sci Med 1999;49:1461-71.

Rappaport, J. (1987). Terms of empowerment/exemplars of prevention: Toward a theory for Community Psychology. American Journal of Community Psychology. 15(2):121-148.

Sanjay, M.T. (1999) Psychological Empowerment: Definition, Measurement, and Validation Canadian Journal of Behavioural Science

Sharma S. Myths and realizations .Journal of Rural and community psychiatry. (7-10).1 (2)2014.

Zimmerman, M. A (1995) Psychological empowerment: Issues and illustrations. American Journal of Community Psychology. 25(5):581-599.

How to cite this article: Sharma S, Gupta A (2017), The Everyday lives and coping Strategies of women in Delhi; Discerning roots of Psychological Empowerment- An Ethnographic Study, International Journal of Indian Psychology, Volume 4, Issue 2, No. 96, ISSN:2348-5396 (e), ISSN:2349-3429 (p), DIP:18.01.198/20170402, ISBN:978-1-365-84232-0

(c) The International Journal of Indian Psychology, ISSN 2348-5396 (e)| ISSN: 2349-3429 (p) | 169 